\title{
Nucleotide Excision Repair Inhibition
}

National Cancer Institute

\section{Source}

National Cancer Institute. Nucleotide Excision Repair Inhibition. NCI Thesaurus. Code

C40662.

Nucleotide Excision Repair (NER) Inhibition involves interference with, or restraint of, the activities that mend DNA regions containing chemical adducts, carcinogenic adducts, or thymine dimers. During NER in eukaryotes, protein complexes detect DNA shape irregularities, induce cuts on each side of the lesion, remove the damaged fragment, and repair the helix by synthesis and ligation. 